\title{
Search for a light charged Higgs boson decaying to a W boson and a CP-odd Higgs boson in trilepton final states in pp collisions at $13 \mathrm{TeV}$ with CMS
}

\author{
Ji Hwan Bhyun ${ }^{a, 1, *}$ \\ ${ }^{a}$ Department of Physics and Astronomy, Seoul National University, Seoul 08826, Republic of Korea \\ E-mail: ji.hwan.bhyun@cern.ch
}

A search for a light charged Higgs boson $\left(\mathrm{H}^{+}\right)$decaying to a $\mathrm{W}$ boson and a CP-odd Higgs boson (A) using trilepton final states (electron-dimuon or trimuon) is presented. The result is based on data from pp collisions at a center of mass energy of $13 \mathrm{TeV}$, recorded by the CMS detector, corresponding to an integrated luminosity of $35.9 \mathrm{fb}^{-1}$. In this search, it is assumed that the $\mathrm{H}^{+}$ boson is produced in decays of top quarks, and the A boson decays to two oppositely charged muons. The first upper limits are set on the combined branching fraction for the decay chain.

40th International Conference on High Energy physics - ICHEP2020

July 28 - August 6, 2020

Prague, Czech Republic (virtual meeting)

${ }^{1}$ On behalf of the CMS Collaboration

* Speaker 
The Higgs boson with a mass around $125 \mathrm{GeV}$ was discovered in 2012 by the ATLAS and CMS Collaborations [1-3], and its observed properties have been consistent with the expectation in the standard model (SM) [4, 5]. However, there are models beyond the SM which have a different Higgs sector compared to the SM, yet are consistent with the experimental results. Two-Higgs-doublet models (2HDM) are an example of such models [6].

In 2HDM, a charged Higgs boson $\left(\mathrm{H}^{+}\right)$and a CP-odd Higgs boson (A) are predicted. The $\mathrm{H}^{+}$boson can be mainly produced in top quark decays ( $\mathrm{pp} \rightarrow \mathrm{t} \overline{\mathrm{t}} \rightarrow \mathrm{b} \overline{\mathrm{b}} \mathrm{W}^{-} \mathrm{H}^{+}$) and decay to $\mathrm{W}$ and $\mathrm{A}$ bosons $\left(\mathrm{H}^{+} \rightarrow \mathrm{W}^{+} \mathrm{A}\right)[7,8]$. The $\mathrm{A}$ boson can decay to an oppositely charged muon pair $\left(\mathrm{A} \rightarrow \mu^{+} \mu^{-}\right.$). A search is performed to investigate this production and decay mode of $\mathrm{H}^{+}$and A bosons. The A boson mass $\left(\mathrm{m}_{\mathrm{A}}\right)$ hypotheses between 15 and $75 \mathrm{GeV}$ and the $\mathrm{H}^{+}$boson mass $\left(\mathrm{m}_{\mathrm{H}^{+}}\right)$hypotheses between $\left(\mathrm{m}_{\mathrm{A}}+85 \mathrm{GeV}\right)$ and $160 \mathrm{GeV}$ are considered. The motivation behind the choice of the range of $m_{H^{+}}$values is based on the mass thresholds of $\left(m_{A}+m_{W}\right)$ and $\left(m_{t}-m_{b}\right)$ for the decay. For the two $\mathrm{W}$ bosons in the decay chain, semileptonic decay channels (WW $\rightarrow \ell v \mathrm{q} \overline{\mathrm{q}}^{\prime}$, $\ell=\mathrm{e}$ or $\mu$ ) are targeted.

The search utilizes a data set of pp collisions at a center of mass energy of $13 \mathrm{TeV}$, corresponding to an integrated luminosity of $35.9 \mathrm{fb}^{-1}$, recorded using the CMS detector [9]. Selected events are required to contain two muons, and one electron or an additional muon. The three leptons should include at least one $\mu^{+} \mu^{-}$pair, and the invariant mass of $\mu^{+} \mu^{-}$pair is required to satisfy $m_{\mu \mu}>12 \mathrm{GeV}$ and $\left|m_{\mu \mu}-91.2\right|>10 \mathrm{GeV}$ for all the $\mu^{+} \mu^{-}$pairs in an event. In addition, the events are required to contain two or more jets, at least one of which is identified as originating from $a b$ quark. The $m_{\mu \mu}$ distribution is analyzed to look for the A boson resonance. The background of the search originates mainly from tt processes that include a nonprompt lepton from $\mathrm{B}$ hadron decays.

No statistically significant evidence of the signal is found, and 95\% confidence level (CL) upper limits are set on the product of branching fractions, $\mathcal{B}_{\text {sig }}=\mathcal{B}\left(\mathrm{t} \rightarrow \mathrm{bH} \mathrm{H}^{+}\right) \mathcal{B}\left(\mathrm{H}^{+} \rightarrow \mathrm{W}^{+} \mathrm{A}\right) \mathcal{B}(\mathrm{A} \rightarrow$

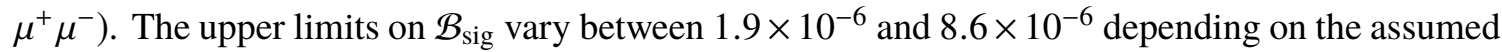
$\mathrm{m}_{\mathrm{H}^{+}}$and $\mathrm{m}_{\mathrm{A}}$ values, as shown in the Fig. 1.

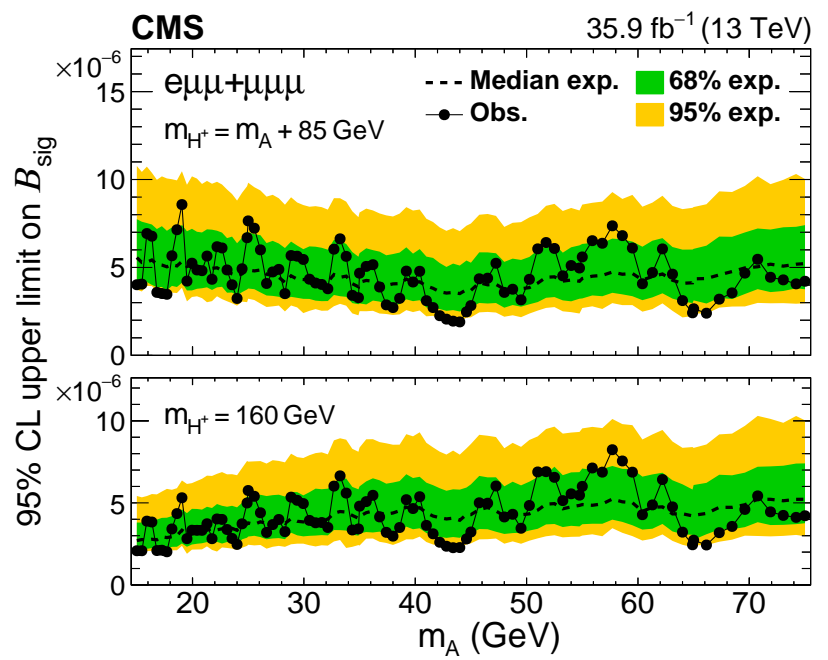

Figure 1: The upper limits at $95 \% \mathrm{CL}$ on $\mathcal{B}_{\text {sig }}=\mathcal{B}\left(\mathrm{t} \rightarrow \mathrm{bH}^{+}\right) \mathcal{B}\left(\mathrm{H}^{+} \rightarrow \mathrm{W}^{+} \mathrm{A}\right) \mathcal{B}\left(\mathrm{A} \rightarrow \mu^{+} \mu^{-}\right)$[10]. In the upper (lower) panel, the $\mathrm{m}_{\mathrm{H}^{+}}$values are assumed to be $\mathrm{m}_{\mathrm{A}}+85 \mathrm{GeV}(160 \mathrm{GeV})$. 
This result can be used to derive upper limits on the product of branching fractions, $\mathcal{B}(\mathrm{t} \rightarrow$ $\left.\mathrm{bH}^{+}\right) \mathcal{B}\left(\mathrm{H}^{+} \rightarrow \mathrm{W}^{+} \mathrm{A}\right)$, at the order of $10^{-2}\left(10^{-3}\right)$ in the type-1 and $2(\mathrm{X}) 2 \mathrm{HDM}$. This is more stringent than the previous results from the CDF experiment, using different decay modes of the A boson $[11,12]$. This is the first search for the decay mode of the $\mathrm{H}^{+}$boson $\left(\mathrm{H}^{+} \rightarrow \mathrm{W}^{+} \mathrm{A} \rightarrow\right.$ $\mathrm{W}^{+} \mu^{+} \mu^{-}$), and it constrains the class of models which predict a significant rate of the production and decay mode of the $\mathrm{H}^{+}$boson.

\section{References}

[1] ATLAS Collaboration, Observation of a new particle in the search for the Standard Model Higgs boson with the ATLAS detector at the LHC, Phys. Lett. B 716 (2012) 1 [1207. 7214].

[2] CMS Collaboration, Observation of a new boson at a mass of $125 \mathrm{GeV}$ with the CMS experiment at the LHC, Phys. Lett. B 716 (2012) 30 [1207.7235].

[3] CMS Collaboration, Observation of a new boson with mass near $125 \mathrm{GeV}$ in pp collisions at $\sqrt{s}=7$ and $8 \mathrm{TeV}, \mathrm{JHEP} 06$ (2013) 081 [1303.4571].

[4] ATLAS and CMS Collaborations, Measurements of the Higgs boson production and decay rates and constraints on its couplings from a combined ATLAS and CMS analysis of the LHC pp collision data at $\sqrt{s}=7$ and 8 TeV, JHEP 08 (2016) 045 [1606.02266].

[5] CMS Collaboration, Combined measurements of Higgs boson couplings in proton-proton collisions at $\sqrt{s}=13 \mathrm{TeV}$, Eur. Phys. J. C 79 (2019) 421 [1809. 10733].

[6] G.C. Branco, P.M. Ferreira, L. Lavoura, M.N. Rebelo, M. Sher and J.P. Silva, Theory and phenomenology of two-Higgs-doublet models, Phys. Rep. 516 (2012) 1 [1106. 0034].

[7] F. Kling, A. Pyarelal and S. Su, Light charged Higgs bosons to AW/HW via top decay, JHEP 11 (2015) 051 [1504.06624].

[8] A. Arhrib, R. Benbrik and S. Moretti, Bosonic decays of charged Higgs bosons in a 2HDM type-I, Eur. Phys. J. C 77 (2017) 621 [1607.02402].

[9] CMS Collaboration, The CMS experiment at the CERN LHC, JINST 3 (2008) S08004.

[10] CMS Collaboration, Search for a light charged Higgs boson decaying to a W boson and a $C P$-odd Higgs boson in final states with e $\mu \mu$ or $\mu \mu \mu$ in proton-proton collisions at $\sqrt{s}=13$ TeV, Phys. Rev. Lett. 123 (2019) 131802 [1905. 07453].

[11] CDF collaboration, Search for charged Higgs bosons from top quark decays in p $\bar{p}$ collisions at $\sqrt{s}=1.96$ TeV, Phys. Rev. Lett. 96 (2006) 042003 [hep-ex/0510065].

[12] CDF collaboration, Search for a very light CP-odd Higgs boson in top quark decays from $p \bar{p}$ collisions at $\sqrt{s}=1.96$ TeV, Phys. Rev. Lett. 107 (2011) 031801 [1104.5701]. 